\title{
Genome-Wide Analysis of NAC Proteins and Characterization of the ATAF Member RcNAC091 in Rose (Rosa Chinensis)
}

\section{Lifang Geng}

Qingdao Agricultural University

Lin Su

Qingdao Agricultural University

Lufeng Fu

Qingdao Agricultural University

Shang Lin

Qingdao Agricultural University

Jianmei Zhang

Yantai Service Center of Forest Resources Monitoring and Protection

Qinghua Liu

Qingdao Agricultural University

Xinqiang Jiang ( $\sim$ jiangxinqiang8@163.com )

Qingdao Agricultural University https://orcid.org/0000-0003-0727-3354

\section{Research Article}

Keywords: Rose, NAC family, Expression pattern, dehydration stress

Posted Date: August 24th, 2021

DOl: https://doi.org/10.21203/rs.3.rs-803598/v1

License: (c) (i) This work is licensed under a Creative Commons Attribution 4.0 International License. Read Full License 


\section{Abstract}

The NAC (NAM, ATAF, and CUC) transcription factors (TFs), as plant-specific transcription regulators, play a critical role in various developmental processes and stress responses. However, knowledge of the NAC TFs in rose (Rosa chinensis), one of the most important horticultural crops, is limited. In this study, 116 NAC genes were identified from the rose genome and classified into 16 subfamilies. The RcNACs were unevenly distributed on the chromosomes. Analysis of the stress-related RcNACs based on the transcriptome data showed differences in the expression patterns in different organs and flower developmental stages and during drought and salt stress in rose leaves and roots. Analysis of the cisregulatory elements (CREs) in the promoter region revealed that stress-related RcNACs possessed three sets of CREs, corresponding to plant growth and development, plant hormonal response, and abiotic and biotic stresses. Reverse transcription-quantitative real-time PCR (RT-qPCR) analysis of 11 representative RcNACs revealed their diverse transcript expression in leaves and roots under abscisic acid (ABA), polyethylene glycol (PEG), and sodium chloride $(\mathrm{NaCl})$ treatments. Furthermore, RcNAC091 silencing verified its role in positively regulating the dehydration stress response. Overall, this study provides helpful insight into stress-related RCNACs and paves the way for stress tolerance via breeding in rose.

\section{Key Message}

The study identified 116 NAC genes in rose. Stress-related RcNACs showed different expression patterns. Silencing RCNAC091, a member of the subfamily ATAF, conferred decreased tolerance to dehydration stress.

\section{Introduction}

The NAC (NAM, ATAF, and CUC) transcription factors (TFs) are plant-specific regulatory proteins that have been extensively studied in the past few years. The NAC obtained the name from the first three members identified, the NAM of Petunia hybrida, the ATAF1/2, and the CUC2 of Arabidopsis thaliana. In general, NAC protein sequences contain two parts; the conserved NAC domain at the N-terminus, consisting of about 150 amino acids, and the highly differentiated transcriptional regulatory region at the C-terminus, regulating transcriptional and downstream gene expression (Mohanta et al. 2020). The conserved NAC domain is divided into five subdomains, named A, B, C, D, and E. Subdomains A, C, and D are highly conserved and correlates with DNA binding. In contrast, subdomains $B$ and $E$ are variable and endow NAC with different functions (Puranik et al. 2012).

In the past few years, NAC TFs have been discovered in various plant species such as Arabidopsis (Ooka et al. 2003), rice (Oryza sativa) (Fang et al. 2008), apple (Malus domestica) (Su et al. 2013), soybean (Glycine max) (Le et al. 2011), wheat (Triticum aestivum) (Guérin et al. 2019), corn (Zea mays) (Shiriga et al. 2014), and banana (Musa acuminata) (Cenci et al. 2014). Many studies have shown that NAC TFs regulate plant growth and development and abiotic stress (salt, drought, heat, mechanical damage) and defense (viruses) responses (Nakashima et al. 2012). Overexpression of RESPONSIVE TO DESICCATION 
26 (RD26) in Arabidopsis increased the sensitivity of plants to abscisic acid (ABA), indicating the role of RD26 in an ABA-dependent stress signaling pathway (Fujita et al. 2004). In rice, the STRESS-RESPONSIVE NAC 1 (SNAC1) overexpression significantly promoted drought resistance and salt tolerance by enhancing ABA sensitivity (Hu et al. 2006). Furthermore, cold, salt, drought, and ABA had induced OsNAC6 in rice, and overexpression of OsNAC6 increased the tolerance of plants to multiple stresses (Ohnishi et al. 2005). Meanwhile, low temperature and water stress had induced SsNAC23 in sugarcane (Saccharum officinarum), which is similar to rice OsNAC6 (Fábio et al. 2005). The MbNAC29 gene from Malus baccata improved the ability to remove reactive oxygen species (ROS) and subsequently enhanced cold and salt tolerance (Han et al. 2020).

Rose (Rosa chinensis), an oranmental perennial shrubs native to China, is widely used outdoors and indoors to improve the aesthetic value of landscapes and has important economic and nutritional values worldwide. The rose flowers also contains various nutrients and fragrances with health benefits. However, roses often encounter adverse environmental conditions that affect their growth characteristics and flower quality. TFs, such as NAC, WRKY, MYB etc have close relationship corresponding to these biological processes. For rose NACs, researchers have identified several key NAC genes, like RhNAC100 (Pei et al. 2013), RhNAC2 (Dai et al. 2012), RhNAC3 (Jiang et al. 2014), and RhNAC31 (Ding et al. 2019), associated with flower development or water deficit stress in rose. Besides, many rose TF families like AP2/ERF (Li et al. 2021) and WRKY (Liu et al. 2019) have been comprehensively studied. However, the comprehensive analysis of NAC family in rose has not been conducted. The genome-wide exploration and expression analysis of the stress-related NAC gene family will provide technical support for stressresistant rose breeding and cultivation.

In this study, 116 RCNAC genes of rose were systematically identified, and the phylogeny, physicochemical properties, gene structure, conserved motifs, and the chromosomal location of these RcNAC genes were investigated. The collinearity relationship of NAC genes among five Rosaceae plant species (rose, apple, strawberry (Fragaria vesca), mei (Prunus mume), and almond (P. dulcis) was also analyzed. The RcNACs were categorized into 16 subfamilies, and 44 stress-related ones were selected for further analysis. The cis-regulatory element of these NAC gene members was analyzed. The expression patterns of these genes in different organs and under different situations, such as floral development, drought, and salt stress, were explored. In particular, 11 representative RcNACs were further selected and analyzed by quantitative reverse-transcription PCR (RT-qPCR). Additionally, the drought-induced RcNAC091 was silenced in rose leaves to investigate its role in regulating tolerance to dehydration stress. Our results strengthen the understanding of RcNACs and provide candidate genes that help adapt to adverse conditions.

\section{Materials And Methods}

Identification of RcNACs 
The complete genome sequence and gff3 files of rose were obtained from GDR (Genome Database for Rosaceae, https://www.rosaceae.org) (Jung et al. 2014). The corresponding data of the NAC family in Arabidopsis were obtained from PlantTFDB (Plant Transcription Factor Database, http://planttfdb.gaolab.org/) (Jin et al. 2017). The Blast Wrapper in TBtools (Chen et al. 2020) was used to retrieve the possible rose NAC sequences based on the reference sequence of the gene family. The rose NAC sequences were compared with the NCBI database (https://www.ncbi.nlm.nih.gov/) to verify the existence of the NAM domain. Then, each RcNAC was checked through the Batch CD-Search tool in the NCBI database to confirm further the results (Aron et al. 2011). Finally, the Pfam database (PF02365) (https ://pfam.xfam.org/) (Finn et al. 2014) detected the conserved NAM domains in the obtained sequences. The characteristics of RcNACs were analyzed using ExPASy (http://web.expasy.org/compute_pi/) (Panu et al. 2012) to predict the protein length, molecular weight $(\mathrm{MW})$, and isoelectric point ( $\mathrm{pl})$.

\section{Phylogenetic, gene structure, and motif analysis}

NAC protein sequences of different species (Arabidopsis, rice, apple, and kiwifruit (Actinidia chinensis) were obtained from Ensembl Plants (https://plants.ensembl.org/index.html) (Kevin et al. 2020), and ten conserved motifs of these proteins were identified using the MEME suite (http://memesuite.org/tools/meme) (Bailey et al. 2009). The exon-intron organization of the RcNAC genes was analyzed using the TBtools. The neighbor-joining method constructed a phylogenetic tree with 1000 bootstrap values using MEGA7 (Molecular Evolutionary Genetics Analysis) (Kumar et al. 2016). Furthermore, the Batch CD-Search tool on the NCBI was used to analyze the domains in all NAC protein sequences.

\section{RcNAC cis-regulatory element (CRE) analysis}

The upstream $1.5 \mathrm{~kb}$ sequences of the selected 44 RcNACs were extracted by TBtools and submitted to the PlantCARE (http://bioinformatics.psb.ugent.be/webtools/plantcare/html/) (Lescot et al. 2020) database to predict the CREs. According to their function, these CREs were categorized into three groups, corresponding to abiotic and biotic stress, phytohormone responsiveness, and plant growth and development. The graph, showing the type and number of cis-elements, was displayed by TBtools and modified by Adobe Illustrator CS5.

\section{RcNAC chromosomal location and collinearity analysis}

The chromosomal location of RcNACs was mapped according to therose genomeinformationobtainedfrom the GDR and visualized by TBtools (Chen et al. 2020). The genome 
data and gene annotation files of apples, strawberries, mei, and almond were downloadedfrom the JGI (Joint Genome Institute, https://genome.jgi.doe.gov/portal/) database to investigate the syntenic relationship among the NAC genes of rose and other plant species. Further, MCScanX (Wang et al. 2012) was used to identify the putative homologous chromosomal regions. To evaluate the evolution of proteincoding regions of the duplicated NAC genes, non-synonymous substitution (Ka), synonymous substitution (Ks), and Ka/Ks were calculated by TBtools.

\section{Heatmap of RcNACs}

The transcript levels of RcNACs in different rose organs (accession number GSE54486) (Yan et al. 2014), during the floral transition (accession number PRJCA000258) (Guo et al. 2017), and in the leaves and roots under drought (accession number PRJNA663119) (Li et al. 2021) or salt stress (accession number SRP128235) (Tian et al. 2018) were retrieved from the transcriptome data in GEO (Gene Expression Omnibus) database,GSA (Genome Sequence Archive) database and SRA (Sequence Read Archive) database. The expression profiles of RCNACs were determined using the FPKM (fragments per kilobase million) values. Expression levels of the RcNACs was illustrated based on the $\log _{2}$ normalized FPKM values using TBtools and visualized by ggplot2 package in $\mathrm{R}$ software.

\section{RT-qPCR analysis}

Further, 11 representative RcNACs were selected for RT-qPCR to validate the expression pattern under abiotic stress. Rose (Rosa hybrida 'sanmatha') tissue culture seedlings with eight compound leaves were transferred to MS medium or MS medium plus $50 \mu \mathrm{M}$ ABA, 10\% PEG 6000 , and $200 \mathrm{mM} \mathrm{NaCl}$ and allowed to grow for five days. The total RNA was extracted from rose leaves and rots using RNAprep Pure Plant Kit (TIANGEN, Beijing, China). Approximately $1 \mu \mathrm{g}$ of total RNA was used for CDNA synthesis using the HiScript II 1st Strand cDNA Synthesis Kit (Vazyme Biotech, Nanjing, China) to remove the genomic DNA. The SYBR qPCR Master Mix (Vazyme Biotech, Nanjing, China) was used to perform the RTqPCR assay on a StepONE Plus system (Applied Biosystems, USA) in a $20 \mu \mathrm{L}$ reaction volume. The reaction mixture consisted of $10 \mu \mathrm{L} 2 \times$ ChamQ Universal SYBR qPCR Master mix, $0.4 \mu \mathrm{L}$ of each primer, 2 $\mu \mathrm{L}$ cDNA, and $7.2 \mu \mathrm{LddH} \mathrm{H}_{2} \mathrm{O}$. The reaction program was as follows: $95^{\circ} \mathrm{C}$ for $15 \mathrm{~s}$, followed by 40 cycles at $95^{\circ} \mathrm{C}$ for $10 \mathrm{~s}, 60^{\circ} \mathrm{C}$ for $20 \mathrm{~s}$, and then $95^{\circ} \mathrm{C}$ for $15 \mathrm{~s}$. Ubiquitin $(R c U B / 2)$ gene was used as an internal control for normalizing all mRNA expression levels; three biological replicates were maintained per treatment, and the gene expression levels were analyzed by $2^{-\Delta \Delta C T}$ method (Livak et al. 2001). The primers used are listed in Table S1.

\section{Silencing RcNAC091 by VIGS}


Virus-induced gene silencing (VIGS) approach was used to silence RcNACO91 (genbank accession number MG878302) in rose leaves. A fragment of approximately $430 \mathrm{bp}$ at the 3 'end of RcNACO91 was cloned into pTRV2 to obtain pTRV2-RcNAC091. The Agrobacterium resuspension cultures containing pTRV1 and pTRV2-RcNACO91 (TRV-RcNACO91), as well as pTRV1 and pTRV2 (TRV, control) were mixed in equal proportions, respectivily. The middle portion of the rose leaves with a punch $(1 \mathrm{~cm})$ of leaf disc was used. VIGS was carried out as described by Jiang et al. (2014). The TRV and TRV-RcNACO91 samples were dehydrated for 0,4 , and $8 \mathrm{~h}$ and then rehydrated for $3 \mathrm{~h}$ under controlled conditions (23-25 ${ }^{\circ} \mathrm{C}, 40 \%-50 \%$ relative humidity, and $100 \mu \mathrm{mol} / \mathrm{m}^{2} / \mathrm{s}$ light intensity). The disc area was monitored at the indicated time points using Image $\mathrm{J}$ (https://imagej.nih.gov/ij). Ion leakage and chlorophyll content were measured after $3 \mathrm{~h}$ of rehydration. Cell death in TRV and TRV-RcNACO91 discs after $3 \mathrm{~h}$ of rehydration was measured by trypan blue staining according to the method by Dai et al. (2018). The ROS in the leaves was stained using 3,3'-diaminobenzidine (DAB) and nitrotetrazolium blue chloride (NBT) as described by Dai et al. (2018). The experiments were repeated at least three times, and each replicate contained five plants.

\section{Results}

\section{Identification of NAC domain proteins in rose}

The HMMER (Prakash et al. 2017) and the Pfam NAC domain (PF02365) were employed to identify the rose NAC family using the protein sequences obtained from the Arabidopsis NAC gene sequence. A total of 123 protein sequences were initially obtained by comparing and searching the rose genome database (https://www.rosaceae.org). A two-way comparison between TBtools (Chen et al. 2020) and the NCBI database was used to detect the NAC domain after removing the redundant sequences. A total of 116 RcNACs were identified and named from RcNAC001 to RcNAC116 according to their chromosome location. These 116 RcNACs were analyzed for their gene location, gene length, molecular weight (Mw), isoelectric point (pl), and subcellular localization (Table S2). The protein length of RcNACs ranged from 70 (RcNAC076) to 714 amino acids (aa, AA) (RcNAC102), with an average of 336 aa (Table S1). The pl ranged from 4.43 (RcNAC107) to 9.78 (RcNAC98), whereas the Mw ranged from $7.87 \mathrm{kDa}$ (RcNAC076) to $79.99 \mathrm{kDa}$ (RcNAC102). Subcellular localization analysis predicted nuclear localization for $49 \%$ of RcNACs and extracellular localization for $51 \%$. These results suggest the crucial roles of RcNACs in the evolution of the rose genome.

\section{Phylogenetic analysis of NAC proteins}

A phylogenic tree was constructed using 736 NAC protein sequences from five species, including rose (116), kiwifruit (156), Arabidopsis (105), apple (228), and Oryza barthii (131). Based on the classification of NAC family proteins in Arabidopsis by Ooka et al. (2003), the NACs were divided into 16 subfamilies, including ANAC011, NAM, NAC1, OSNAC7, OSNAC8, TIP, NAC2, ONAC22, ANAC001, SEUN5, ONAC003, 
TERN, ANAC063, ATAF, NAP, and Unclassified. The ANAC001 subfamily was the largest with 23 members ( $20 \%$ of the total RcNACs) in rose, followed by the NAM subfamily containing 11 RcNACs; OSNAC8 and ANAC063 subfamilies were the minor categories with only one member each. However, no plant species had all the subfamilies, and no OSNAC8 member was found in Arabidopsis (Table S3). Furthermore, the percentage of each subfamily was different among the plant species. For example, monocots like $O$. barthii (19\%) showed a more significant percentage of the TIP subfamily than the dicots likeroses(4\%), kiwifruit(6\%), Arabidopsis(7\%), andapple(4\%) (Fig. 1). These findings indicate potential differences in levels of gene duplication or loss between monocots and dicots that might have occurred during evolution.

\section{Gene structure and motif analysis of RcNACs}

Further, the conserved motifs, exon-intron structure, and phylogeny were analyzed to understand better the structural diversity in rose NAC proteins (Fig. 2). A total of ten conserved motifs, mainly localized in the N-terminus, were identified for NAC proteins (Fig. S1). Motifs 1-7 were highly conserved and distributed in most rose NACs ( 60\%), while motifs 8-10 were distributed in a few subfamilies (ANAC001 and Unclassified). Most of the conserved motifs of rose NACs of the subfamilies, such as ANAC011, TIP, and NAC2, were similar, indicating a relatively close evolutionary relationship. However, the conserved motifs of RcNACs in subfamilies ANAC001, ONAC003, and ANAC063 were different, indicating a rather distant evolutionary relationship.

Meanwhile, the exon-intron organization showed that the intron number was diverse among RcNACs, ranging from one to nine (Fig. 2). All 116 RcNACs consisted of one or more exons. The maximum number of exons (seven) was found in RcNAC102 of the TIP subfamily, and the average number of exons in the 116 RCNACs was four. Furthermore, the genes in the same subfamily had similar gene structures, with similar intron and exon arrangement and numbers, indicating highly conserved biological functions of these genes.

\section{Chromosome localization and collinearity analysis of RcNACs}

Chromosome mapping revealed that the RcNACs were unevenly distributed within the rose genome composed of seven chromosomes. Among them, chromosome 1 contained the maximum RcNACs (31, $26.7 \%)$, followed by chromosome $5(21,18.1 \%)$; the least number of RcNACs was on chromosome 4 (6, 5.2\%; Fig. 3A).

Furthermore, a collinearity analysis was performed using NACs from rose and four other Rosaceae plants, including apple, strawberry, mei, and almond. As shown in Fig. 3B, the collinearity between the NACs in rose and apple (142) was significantly higher than the other three species (mei, 79; strawberry, 66; and 
almond, 40). The RCNACs on chromosomes 5 and 7 had significantly more collinear gene pairs than the other chromosomes. Less than ten collinear gene pairs were identified between rose and almond, which may be related to the phylogenetic relationship between species (Fig. 3B). These results suggest that few RcNACs were possibly produced by gene and segmental duplication events, which probably played a major driving force for RCNAC gene evolution.

\section{Relative transcript levels of stress-related RcNACs in different transcriptome datasets}

NAC proteins play various roles in plant growth, development, and stress response. In this study, 44 stress-related RcNACs belonging to eight subfamilies (ATAF, NAC2, TIP, SNUE5, NAM, NAP, ONAC022, ONAC003) were selected for further analysis. Sequence homology of all these genes with already reported stress-related NACs indicated their close association with biotic or abiotic stresses (Shao et al. 2015). The expression levels of 44 RcNACs at the three stages of the rose flower transition (vegetative meristem, pre-floral meristem, floral meristem) and in the six organs (prickle, stamen, leaf, pistil and ovary, stem, and root) based on public RNA-seq data are shown in Fig. 4A. The transcript levels of RcNACs of the same or different subfamilies were highly variable among the organs or the transition stages, suggesting their multiple functions in growth and development. Interestingly, the expression levels of four NAC2s (RcNAC008, RcNAC086, RcNAC009, RcNAC088), three ATAFs (RcNAC034, RcNAC061, RcNAC091), two TIPs (RcNAC031, RCNAC102), and one SNEU5 (RcNAC056) in the various organs and flower transition processes were relatively high, demonstrating their roles in rose growth and development.

To further gain insight into the putative functions of these 44 RcNACs in response to abiotic stress, their expression patterns under drought stress in leaves (no drought, NDL; mild drought stress, MDL; severe drought stress, SDL) and roots (NDR, MDR, SDR) and salt stress in leaves (TL0, TL2, TL4, TL7, TL9) and roots (TR0, TR2, TR4) were evaluated (Fig. 4B). Overall, the expression levels of the RcNACs from eight subfamilies were different. These genes showed dramatic changes under various degrees of drought or salt stress in rose leaves and roots, further confirming their roles in response to abiotic stress. Meanwhile, 11 RcNACs of the same clade showed induced expression under drought or salt stress in rose leaves and roots. Specifically, three ATAFs (RcNAC034, RcNAC069, RcNAC091) showed higher expression levels under drought or salt stress. These expression profiles demonstrated functional convergence and differences of the RCNACs, which probably occurred during evolution.

\section{Analysis of the cis-regulatory element (CRE) in stress-related RcNACs promoter}

To analyze the regulation of stress-related RcNAC gene expression, the CREs of its promoter, a region of approximately $1.5 \mathrm{~kb}$ upstream of the initial transcript site, were tested. Li et al. (2021) classified the 
promoters into three categories based on functions, corresponding to plant growth and development (Box4, G-box, CAT-box, AE-box, GT1-motif, GATA-motif, l-box), plant hormone response (ABRE, CGTCAmotif, ERE, TGACG-motif, GARE-motif, TGA-element), and abiotic and biotic stress (TC-rich repeats, MBS, LTR, WUN-motif, W box, WRE3, STRE, MYB, MYC, ARE, Myb-binding site, TCT-motif).These CREs were randomly dispersed among the different RcNACs promoters. RcNACO34 had the most significant number of CREs (57), followed by RCNACO91 (52), while RCNACO81 had the least number of CREs (6). Among the different types of CREs, abiotic and biotic stress-related CREs, including MYB, MYC, Box4, G box, and ABRE, were significantly more than the other CREs (Fig. 5); 31 out of 44 RcNACs contained ABRE elements, suggesting RcNACs roles in regulating the response to abiotic stresses through ABA-dependent pathway.

\section{Expression of selected RcNACs}

According to the transcriptome expression and CREs analysis, 11 representative genes (RCNACO09, RcNAC031, RcNAC034, RcNACO56, RcNAC059, RcNAC062, RcNAC069, RcNAC086, RcNAC088, RcNAC091, RcNAC102) belonging to six subfamilies of the same clade (Fig. 4B) were selected for further analysis using RT-qPCR under ABA, PEG, and NaCl treatments (abiotic stress) in rose leaves and roots. As shown in Fig. 6, members of the different subfamilies showed differential expression patterns. RcNACO34 and $R C N A C 069$ showed upregulated expression levels when treated with ABA in leaves and roots, while RcNACO59 and RcNACO31 did not. PEG upregulated RcNACO56 in leaves, and ABA upregulated in roots. The expression levels of both RcNACO31 and RcNAC102 showed no change under ABA, PEG, and $\mathrm{NaCl}$ treatments. However, RcNACO91 showed upregulated expression levels when treated with ABA, PEG, and $\mathrm{NaCl}$. These expression profiles of RcNACs from different subfamilies indicated conserved or diverse roles in rose leaves and roots in response to external cues.

\section{Silencing RcNAC091 decreases tolerance to dehydration stress}

RcNAC091 showed an stongly induced expression pattern in response to abiotic stress (Fig. 6). Therefore, VIGS was used to silence the selected RcNAC091 for functional analysis. As shown in Fig.7, about $40 \%$ of RcNACO91 was silenced in rose (Fig. 7A). No obvious difference in phenotype was observed between TRV and TRV-RcNACO91 under dehydration $0 \mathrm{~h}$. However, when dehydrated for $4 \mathrm{~h}$ and $8 \mathrm{~h}$, discs of TRVRcNACO91 showed more wilting symptoms than TRV controls (Fig. 7B). The discs areas of TRVRcNAC091 under dehydration for $4 \mathrm{~h}$ and $8 \mathrm{~h}$ and rehydration for $3 \mathrm{~h}$ were $510 \mathrm{~mm}^{2}, 395 \mathrm{~mm}^{2}, 556 \mathrm{~mm}^{2}$, respectively, which were significantly lower than TRV controls at the indicated time points $\left(574 \mathrm{~mm}^{2}, 459\right.$ $\mathrm{mm}^{2}, 615 \mathrm{~mm}^{2}$ ) (Fig. 7C). Further, TRV-RcNAC091 samples after rehydration for $3 \mathrm{~h}$ showed lower chlorophyll content (Fig. 7D), with a higher ion leakage than TRV controls (Fig. 7E). Furthermore, TB, DAB, and NBT staining used to investigate the cell death rate and ROS accumulated levels revealed deeper 
staining for TRV-RCNAC091 than TRV controls under rehydration for $3 \mathrm{~h}$ (Fig. 7F), indicating severely damaged cell membranes. These results suggest that RcNACO91 positively regulates dehydration stress response in rose plants.

\section{Discussion}

TFs regulate various important cellular processes in plants, especially when subjected to biotic and abiotic stresses. NAC family is one of the largest TFs that play critical roles in plant growth, development, and stress response (Wang et al. 2013). Genome-wide analysis of NAC has been carried out in Arabidopsis (Ooka et al. 2003), rice (Nuruzzaman et al. 2010), pearl millet (Dudhate et al. 2021), soybean (So and Lee, 2019), and various other species. Nevertheless, studies on the rose NACs are limited. The present study identified 116 NAC genes containing NAM domains from the rose genome. The number of NAC TFs varies between 30 and 228 among different plant species. Researchers have identified 33 TFs in Physcomitrella patens (Bo et al. 2014), 60 in rape (Brassica napus)(Wang et al. 2015), 105 in Arabidopsis (Ooka et al. 2003), and 228 in apple (Su et al. 2013). The genome sizes of these species are also different (rape, $630 \mathrm{Mb}$; Arabidopsis, $116 \mathrm{Mb}$; apple, $682 \mathrm{Mb}$ ); however, the number of NAC family members was not directly related to the size of the genome. These data suggest that NAC proteins numbers have extensively expanded with plant evolution.

Furthermore, the study found differences in the number of NAC subfamily members among rose and other species, indicating the evolutionary differences among plant species. The NAC proteins were categorized into 16 subfamilies; however, NAC genes of all plant species cannot be divided into 16 subfamilies (Fig. 1, Table S2). In A. chinensis, no members of the OSNAC8 subfamily were identified, indicating a loss of extrons/introns during the evolutionary process.

Further analysis showed that although the $\mathrm{pl}, \mathrm{Mw}$, and length of RcNAC proteins were different, the gene composition was similar, indicating a relatively conserved gene structure. About $83 \%$ of the RcNACs contained more than six motifs, while the subfamily ONAC003 contained only four motifs (Fig. 2). Meanwhile, the subfamily ANAC001 contained motif 9 and 10 that others lacked. Besides, genes from the same subfamilies alsp had identical or similar exon-intron compositions. The differences in motifs distribution pettern between RcNAC subfamilies indicate that many genes have evolved. In addition, the composition and distribution of conserved motifs supported the phylogenetic relationship and classification of RcNACs. Previous research has shown that the conserved motifs play an important role (Singh et al. 2002). Most RcNACs contained motifs 1-7, while few contained motifs $8-10$, indicating related functions of these NACs. Although the number of introns ranged from 1 to 8 , about $74 \%$ of the 116 RcNACs had only two introns or even less, consistent with tobacco (Li et al. 2018), pepper (Diao et al. 2018), and Coffea canephora (Dong et al. 2019). Differences in intron number among thee subfamilies suggest the occurrence of alternative splicing, which might help plants to adapt to environmental changes, especially abiotic stress (Laloum et al. 2018). 
RcNACs were found unevenly distributed on seven rose chromosomes, and the numbers in each chromosome ranged from 7 to 31 (Fig. 3). The number of RcNACs on the shortest chromosome was not the least, and the number on the longest chromosome was not the highest, suggesting no positive correlation between the length of the chromosome and the number of RcNACs. Few genes such as RcNACO03, RcNACO04, RcNACO05, RcNACO06, and RcNACO07 were located as clusters on chromosomes, which suggest that the genes may work together through protein isoforms. The collinearity analysis identified only seven homologous gene pairs between rose and almond, while more were identified between rose and strawberry, followed by plum. These observations suggest a close association of rose with apple and strawberry.

Based on the phylogenetic relationship (Fig. 2) and previous reports (Shao et al. 2015), 44 stress-related RcNACs from eight subfamilies were selected for further analysis. Investigation of $1.5 \mathrm{~kb}$ region upstream of stress-related RcNACs promoter revealed that these genes possessed different stress response CREs, such as ABRE, MBS, MYB, and MYC, which may play important roles in response to various stresses. In particular, three RcNACs (RcNAC034, RcNAC069, RcNAC097) of the ATAF subfamily contained more than 10 ABRE elements, proving their outstanding role in responding to environmental stimuli through the ABAdependent pathway (Yoshihiro et al. 2003). Furthermore, 38 out of 44, and 37 out of 44, contained MYB and MYC CREs in their promoter regions, respectively. These two CREs are the binding sites of the MYB and MYC TFs, which can resist biotic and abiotic stresses and regulate plant growth and development (Dar et al. 2017).

Gene expression profiles are often correlated with their biological function. Analysis of the stress-related RcNACs based on transcriptome data revealed that not all RcNACs have a clear response to major abiotic stresses, such as drought and salt stress. The expression profiles of different RcNACs of the same subfamily were different (Fig. 4). The subfamily ATAF is the most studied NAC TFs in response to biotic and abiotic stresses in Arabidopsis (Hu et al. 2006) and Populus euphratica (Lu et al. 2018). Three rose ATAF genes (RcNAC034, RcNAC069, RcNAC091) were found induced under drought and salt (Fig. 5), consistent with RT-qPCR results (Fig. 6). In addition, the response of the other seven subfamily genes to drought and/or salt stress was not noticeable. Interestingly, these genes, induced under drought and/or salt, showed higher expression levels than other genes during flower differentiation stages or different organs. The analysis revealed that RcNACs (RcNAC009, RcNAC056, RcNAC088, RcNAC091) were highly expressed during different developmental stages and in various tissues, suggesting their role in growth and development in rose. To further verify the accuracy of the transcriptome data, 11 genes with high expression levels in response to drought and salt stress were analyzed by RT-qPCR. The RT-PCR results were slightly different from the transcriptome data, probably due to plant material size and processing method differences. The detailed role of NAC proteins in response to abiotic stresses remains to be elucidated.

Members of the ATAF subfamily have been shown to regulate plant responses during abiotic and biotic stresses. A few members from the ATAF subfamily have been well-characterized. For example, rice OsNAC6 (Ohnishi et al. 2005), Haloxylon ammodendron HaNAC1 (Gong et al. 2020), and Miscanthus 
Iutarioriparius MINAC5 (Yang et al. 2005) enhanced drought tolerance. In contrast, certain other members of the ATAF subfamily negatively regulate the response to abiotic stress. For instance, Arabidopsis ATAF1 negatively regulated drought stress (Lu et al. 2007), while soybean GmNAC2 acted as a negative regulator during abiotic stress and participated in the ROS signaling pathway (Jin et al. 2013). In this study, RcNAC091, a member of subfamily ATAF, demonstrated a positive role in response to drought stress. Silencing of RcNACO91 in rose leaves resulted in a decreased tolerance to dehydration, with higher ion leakage and lower chlorophyll content and disc area (Fig. 7). These findings indicate that the role of ATAF members in mediating stress tolerance is diverse and sophisticated among the various plant species.

\section{Conclusions}

In the present study, 116 RcNAC genes were identified from the rose genome and analyzed for their evolutionary relationship, gene structure, and conserved motifs. We have analyzed of the stress-related RcNACs CREs and their expression profiles. Further, the expression profiles of 11 representative RcNACs under ABA, PEG, and NaCl treatments were analyzed by RT-qPCR, and drought-induced RCNACO91, a member of the ATAF subfamily, was identified. Finally, the silencing of RCNACO91 showed decreased of tolerance to dehydration. The present study improves the understanding of the role of NACs and provides a candidate stress-resistant NAC gene in rose.

\section{Declarations}

\section{Acknowledgements}

The research was supported by the National Key Research and Development Program of China (No. 2018YFD1000400) and Innovative Program for Graduate Student of Qingdao Agricultural University (No. QNYCX21085). The authors would like to thank TopEdit (www.topeditsci.com) for linguistic assistance during preparation of this manuscript.

\section{Authors' contributions}

JX conceived and designed the experiments; GL, SL, and FL carried out the experiments; GL and LS conducted the data analysis. JX and GL wrote the manuscript; ZJ and LQ revised the manuscript and contributed the plant material. All authors read and approved the final manuscript.

\section{Competing interests}

The authors declare that they have no known competing financial interests or personal relationships that could have appeared to influence the work reported in this paper.

\section{References}


1. Aron MB, Lu S, Anderson JB, Farideh C, Derbyshire MK, Carol D, Fong JH, Geer LY, Geer RC, Gonzales NR, Gwadz M, Hurwitz DI, Jackson JD, Ke Z, Lanczycki CJ, Lu F, Marchler GH, Mullokandov M, Omelchenko MV, Robertson CL, Song JS, Thanki N, Yamashita RA, Zhang D, Zhang N, Zheng C, Bryant SH (2011) CDD: a conserved domain database for the functional annotation of proteins. Nucleic Acids Res 39:D225-D229. https://doi.org/10.1093/nar/gkq1189

2. Bailey TL, Boden M, Buske FA, Frith M, Grant CE, Clementi L, Ren J, Li WW, Noble WS (2009) MEME SUITE: tools for motif discovery and searching. Nucleic Acids Res 37:W202-8. https://doi.org/10.1093/nar/gkp335

3. Prakash A, Jeffryes M, Bateman A, Finn R (2017) The hmmer web server for protein sequence similarity search. Curr Protoc Bioinformatics 60:3.15.1-3.15.23. https://doi.org/10.1002/cpbi.40

4. Bo X, Misato O, Masatoshi Y, Kiminori T, Mayumi W, Mayuko S, Minoru K, Yoshimi N, Ryosuke S, Yuji Hi, Takashi M, Tetsuya K, Arata Y, Ko K, Mitsuyasu H, Taku D (2014) Contribution of NAC transcription factors to plant adaptation to land. Sci 343:1505-1508. https://doi.org/10.1126/science.1248417

5. Cenci A, Guignon V, Roux N, Rouard M (2014) Genomic analysis of NAC transcription factors in banana (Musa acuminata) and definition of NAC orthologous groups for monocots and dicots. Plant Mol. Biol. Rep 85:63-80. https://doi.org/10.1007/s11103-013-0169-2

6. Chen C, Chen H, Zhang Y, Thomas H, Frank M, He Y, Xia R (2020) TBtools: an integrative toolkit developed for interactive analyses of big biological data. Mol Plant 13. https://doi.org/10.1101/289660

7. Dai F, Zhang C, Jiang X, Kang M, Yin X, Lü P, Zhang X, Zheng Y, Gao J (2012) RhNAC2 and RhEXPA4 are involved in the regulation of dehydration tolerance during the expansion of rose petals. Plant Physiol 160:2064-2082. https://doi.org/10.1104/pp.112.207720

8. Dai W, Wang M, Gong X, Liu JH (2018) The transcription factor FcWRKY40 of Fortunella crassifolia functions positively in salt tolerance through modulation of ion homeostasis and proline biosynthesis by directly regulating SOS2 and P5CS1 homologs. New Phytol 219:972-989. https://doi.org/10.1111/nph.15240

9. Dar NA, Amin I, Wani W, Wani SA, Shikari AB, Wani SH, Masoodi KZ (2017) Abscisic acid: A key regulator of abiotic stress tolerance in plants. Plant Gene 11:106-111. https://doi.org/10.1016/j.plgene.2017.07.003

10. Diao W, John S, Wang S, Liu J, Pan B, Guo G, Ge W, Mohammad D, Ali SH (2018) Genome-wide analyses of the NAC transcription factor gene family in pepper (Capsicum annuum $L$.): chromosome location, phylogeny, structure, expression patterns, cis-elements in the promoter, and interaction network. Int J Mol Sci 19:1048-1061. https://doi.org/10.3390/ijms19041028

11. Ding A, Li S, Li W, Hao Q, Wan X, Wang K, Liu Q, Liu Q, Jiang Q (2019) RhNAC31, a novel rose NAC transcription factor, enhances tolerance to multiple abiotic stresses in Arabidopsis. Acta Physiol Plant 41. https://doi.org/10.1007/s11738-019-2866-1 
12. Dong X, Jiang Y, Yang Y, Xiao Z, Bai X, Gao J, Tan S, Yoonkang H, Hao S, He F (2019) Identification and expression analysis of the NAC gene family in coffea canephora. Agron 9:670. https://doi.org/10.3390/agronomy9110670

13. Dubois A, Remay A, Raymond O, Balzergue S, Chauvet A, Maene M, Pe'crix Y, Yang S, Jeauffre J, Thouroude T, Boltz V, Martin M, Janczarski S, Legeai F, Renou JP, Vergne P, Bris M, Foucher F, Bendahmane M (2011) Genomic approach to study floral development genes in Rosa sp. PLoS One 6:e28455. https://doi.org/10.1371/journal.pone.0028455

14. Dudhate A, Shinde H, Yu P, Tsugama D, Gupta SK, Liu S, Takano T (2021) Comprehensive analysis of NAC transcription factor family uncovers drought and salinity stress response in pearl millet (Pennisetum glaucum). BMC Genom. https://doi.org/10.21203/rs.3.rs-37445/v3

15. Fábio TN, Paulo SS, Sandra RC, Jorge HF, De Rosa V, Pompermayer P, Arruda P (2005) SsNAC23, a member of the NAC domain protein family, is associated with cold, herbivory and water stress in sugarcane. Plant Sci 169:93-106. https://doi.org/10.1016/j.plantsci.2005.03.008

16. Fang Y, You J, Xie K, Xie W, Xiong L (2008) Systematic sequence analysis and identification of tissuespecific or stress-responsive genes of NAC transcription factor family in rice. Mol Genet Genomics 280:547-563. https://doi.org/10.1007/s00438-008-0386-6

17. Finn RD, Alex B, Jody C, Penelope C, Eberhardt RY, Eddy SR, Andreas H, Kirstie H, Liisa H, Jaina M (2014) Pfam: the protein families database. Nucleic Acids Res 42:D222-D230. https://doi.org/10.1093/nar/gkt1223

18. Fujita M, Fujita Y, Maruyama K, Seki M, Hiratsu K, Ohme-Takagi M, Tran LP, Yamaguchi-Shinozaki K, Shinozaki K (2004) A dehydration-induced NAC protein, RD26, is involved in a novel ABA-dependent stress-signaling pathway. Plant J 39:863-876. https://doi.org/10.1111/j.1365-313X.2004.02171.X

19. Gong L, Zhang H, Liu X, Gan X, Nie F, Yang W, Zhang L, Chen Y, Song Y, Zhang H (2020) Ectopic expression of HaNAC1, an ATAF transcription factor from Haloxylon ammodendron, improves growth and drought tolerance in transgenic Arabidopsis. Plant Physiol Bioch 151:535-544.

https://doi.org/10.1016/j.plaphy.2020.04.008

20. Guérin C, Roche J, Allard V, Ravel C, Mouzeya S, Bouzidi MF (2019) Genome-wide analysis, expansion and expression of the NAC family under drought and heat stresses in bread wheat ( $\underline{T}$. aestivum L.). PLoS One 14:e0213390. https://doi.org/10.1371/journal.pone.0213390

21. Guo X, Yu C, Luo L, Wan H, Zhen N, Xu T, Tan J, Pan H, Zhang Q (2017) Transcriptome of the floral transition in Rosa chinensis 'Old Blush'. PMC. https://doi.org/10.1186/s12864-017-3584-y

22. Han D, Du M, Zhou Z, Wang S, Li T, Han J, Xu T, Yang G (2020) An NAC transcription factor gene from Malus baccata, MbNAC29, increases cold and high salinity tolerance in Arabidopsis. In Vitro Cell DevPI 56:588-599. https://doi.org/10.1007/s11627-020-10105-9

23. Hu H, Dai M, Yao J, Xiao B, Xiong L (2006) Overexpressing a NAM, ATAF, and CUC (NAC) transcription factor enhances drought resistance and salt tolerance in rice. Proc. Natl. Acad. Sci. U.S.A 103:1298712992. https://doi.org/10.1073/pnas.0604882103 
24. Jiang X, Zhang C, Lü P, Jiang G, Liu X, Dai F, Gao J (2014) RhNAC3, a stress-associated nac transcription factor, has a role in dehydration tolerance through regulating osmotic stress-related genes in rose petals. Plant Biotechnol. J 12:38-48. https://doi.org/10.1111/pbi.12114

25. Jin $H$, Huang $F$, Cheng $H$, Song $H, Y u D$ (2013) Overexpression of the GmNAC2 gene, an NAC transcription factor, reduces abiotic stress tolerance in tobacco. Plant Mol. Biol. Rep 31:435-442. https://doi.org/10.1007/s11105-012-0514-7

26. Jin J, Tian F, Yang D, Meng Y, Kong L, Luo J, Gao G (2017) PlantTFDB 4.0: toward a central hub for transcription factors and regulatory interactions in plants. Nucleic Acids Res 45:D1040-D1045. https://doi.org/10.1093/nar/gkw1328

27. Jung $\mathrm{S}$, Ficklin SP, Lee T, Cheng CH, Blenda A, Zheng P, Yu J, Bombarely A, Cho I, Ru S, Evans K, Peace C, Abbott AG, Mueller LA, Olmstead MA, Main D (2014) The genome database for rosaceae (GDR): year 10 update. Nucleic Acids Res 42:D1237-D1244. https://doi.org/10.1093/nar/gkt1012

28. Kevin LH, Bruno C, Nishadi DS, Gareth M, Wasiu A, James A, Jorge A, Matthieu B, Dan MB, Lahcen C, Manuel C, Marc C, Mikkel C, Carla C, Alayne C, Paul D, Silvie F, Astri G, Nancy G, Laurent G, Parul G, Kim EH, Erin H, Sarah EH, Pankaj J, Sophie HJ, Paul JK, Nick L, Uma M, Thomas M, Mark DM, Ben M, Matthieu M, Guy N, Sushma N, Andrew O, Irene P, Mateus P, Michael P, Helder P, Emily P, Justin P, Marc R, Matthew R, Vasily S, Daniel MS, Joshua S, Marcela KT, Stephen JT, Martin U, Sharon W, Doreen W, Gary W, Andrew DY, Paul F (2020) Ensembl Genomes 2020-enabling non-vertebrate genomic research. Nucleic Acids Res 48:D689-D695. https://doi.org/10.1093/nar/gkz890

29. Kumar S, Stecher G, Tamura K (2016) MEGA7: molecular evolutionary genetics analysis version 7.0 for bigger datasets. Mol. Biol. Evol 33:1870-1874. https://doi.org/10.1093/molbev/msw054

30. Laloum T, Martín G, Duque P (2018) Alternative splicing control of abiotic stress responses. Trends in Plant Sci 23:P140-150. https://doi.org/10.1016/j.tplants.2017.09.019

31. Le DT, Nishiyama R, Watanabe Y, Mochida K, Yamaguchi-Shinozaki K, Shinozaki K, Tran LP (2011) Genome-wide survey and expression analysis of the plant-specific NAC transcription factor family in soybean during development and dehydration stress. DNA Res 18:263-276.

https://doi.org/10.1093/dnares/dsr015

32. Lescot M, Déhais P, Thijs G, Marchal K, Moreau Y, Van de Peer Y, Rouzé P, Rombauts S (2020) PlantCARE, a database of plant cis-acting regulatory elements and a portal to tools for in silico analysis of promoter sequences. Nucleic Acids Res 30:325-327.

https://doi.org/10.1093/nar/30.1.325

33. Li W, Geng Z, Zhang C, Wang K, Jiang X (2021) Whole-genome characterization of Rosa chinensis AP2/ERF transcription factors and analysis of negative regulator RCDREB2B in Arabidopsis. BMC Genom 22:90. https://doi.org/10.1186/s12864-021-07396-6

34. Li W, Fu L, Geng Z, Zhao X, Liu Q, Jiang X (2021) Physiological characteristic changes and full-length transcriptome of rose (Rosa chinensis) roots and leaves in response to drought stress. Plant Cell Physiol 61:2153-2166. https://doi.org/10.1093/pcp/pcaa137. 
35. Li W, Li X, Chao J, Zhang Z, Wang W, Guo Y (2018) NAC family transcription factors in tobacco and their potential role in regulating leaf senescence. SRA. https://doi.org/10.3389/fpls.2018.01900

36. Liu X, Li D, Zhang S, Xu Y, Zhang Z (2019) Genome-wide characterization of the rose (Rosa chinensis) WRKY family and role of RcWRKY41 in gray mold resistance. BMC Plant Biol 19:522. https://doi.org/10.1186/s12870-019-2139-6

37. Livak KJ, Schmittgen DT (2001) Analysis of relative gene expression data using real-time quantitative PCR and the $2-\triangle \Delta C T$ method. Methods 25:402408. https://doi.org/10.1006/meth.2001.1262

38. Lu X, Zhang X, Duan H, Lian C, Liu C, Yin W, Xia X (2018) Three stress-responsive NAC transcription factors from Populus euphratica differentially regulate salt and drought tolerance in transgenic plants. Physiol Plant 162:73-97. https://doi.org/10.1111/ppl.12613

39. Lu P, Chen N, Rui A, Su Z, Qi B, Ren F, Chen J, Wang X (2007) A novel drought-inducible gene, ATAF1, encodes a nac family protein that negatively regulates the expression of stress-responsive genes in Arabidopsis. Plant Mol. Biol. Rep 63:289-305. https://doi.org/10.1007/s11103-006-9089-8

40. Mohanta TK, Yadav D, Khan A, Hashem A, Tabassum B, Khan AL, Abd_Allah EF, Al-Harrasi A (2020) Genomics, molecular and evolutionary perspective of NAC transcription factors. PLoS One 15:e0231425. https://doi.org/10.1371/journal.pone.0231425

41. Nakashima K, Takasaki H, Mizoi J, Shinozakicd K, Yamaguchi-Shinozakiab K (2012) NAC Transcription Factors in Plant Abiotic Stress Responses. Biochim Biophys Acta Biomembr 1819:97103. https://doi.org/10.1016/j.bbagrm.2011.10.005

42. Nuruzzaman M, Manimekalai R, Sharoni AM, Satoh K, Kondoh H, Ooka H, Kikuchi S (2010) Genomewide analysis of NAC transcription factor family in rice. Gene 465:30-44. https://doi.org/10.1016/j.gene.2010.06.008

43. Ohnishi T, Sugahara S, Yamada T, Kikuchi K, Yoshiba Y, Hirano HY, Tsutsumi, N (2005) OsNAC6, a member of the NAC gene family, is induced by various stresses in rice. Genes Genet Syst 80:135-139. https://doi.org/10.1266/ggs.80.135

44. Ooka H, Satoh K, Doi K, Nagata T, Otomo Y, Murakami K, Matsubara K, Osato N, Kawai J, Carninci P, Hayashizaki Y, Suzuki K, Kojima K, Takahara Y, Yamamoto K, Kikuchi S (2003) Comprehensive analysis of NAC family genes in Oryza sativa and Arabidopsis thaliana. DNA Res 10:239-247. https://doi.org/10.1093/dnares/10.6.239

45. Panu A, Manohar J, Konstantin A, Delphine B, Gabor C, Edouard C, Séverine D, Volker F, Arnaud F, Elisabeth G, Aurélien G, Céline H, Vassilios I, Dmitry K, Robin L, Sébastien M, Khaled M, Nicole R, Grégoire R, loannis X, Heinz S (2012) ExPASy: SIB bioinformatics resource portal. Nucleic Acids Res 40هW597-W603. https://doi.org/10.1093/nar/gks400

46. Pei H, Ma N, Tian J, Luo J, Chen J, Li J, Zheng Y, Chen X, Fei Z, Gao J (2013) An NAC transcription factor controls ethylene-regulated cell expansion in flower petals. Plant Physiol 163:775-791. https://doi.org/10.1104/pp.113.223388 
47. Puranik S, Sahu PP, Srivastava P, Prasad M (2012) NAC proteins: regulation and role in stress tolerance. Trends in Plant Sci 17:369-81. https://doi.org/10.1016/j.tplants.2012.02.004

48. Shao $\mathrm{H}$, Wang $\mathrm{H}$, Tang $X$ (2015) NAC transcription factors in plant multiple abiotic stress responses: progress and prospects. Front. Plant Sci 6:902. https://doi.org/10.3389/fpls.2015.00902

49. Shiriga K, Sharma R, Kumar K, Yadavc SK, Hossaina F, Thirunavukkarasua N (2014) Genome-wide identification and expression pattern of drought-responsive members of the NAC family in maize. Meta Gene 2:407-417. https://doi.org/10.1016/j.mgene.2014.05.001

50. Singh KB, Foley RC, Luis Oñate-Sánchez (2002) Transcription factors in plant defense and stress responses. Curr. Opin. Plant Biol 5:430-436. https://doi.org/10.1016/S1369-5266(02)00289-3

51. So HA, Lee JH (2019) NAC Transcription Factors from Soybean (Glycine max L.) Differentially Regulated by Abiotic Stress. J. Plant Biol 62:147-160. https://doi.org/10.1007/s12374-018-0285-2

52. Su H, Zhang S, Yuan X, Chen C, Wang X, Hao Y (2013) Genome-wide analysis and identification of stress-responsive genes of the NAM-ATAF1,2-CUC2 transcription factor family in apple. Plant Physiol Bioch 71:11-21. https://doi.org/10.1016/j.plaphy.2013.06.022

53. Tian X, Wang Z, Zhang Q, Ci H, Wang P, Yu L, Jia G (2018) Genome-wide transcriptome analysis of the salt stress tolerance mechanism in Rosa chinensis. PLoS One 13:e0200938.https://doi.org/10.1371/journal.pone.0200938

54. Wang B, Guo X, Wang C, Ma J, Niu F, Zhang H, Yang B, Liang W, Han F, Jiang Y (2015) Identification and characterization of plant-specific NAC gene family in canola (Brassica napus $L$.) reveal novel members involved in cell death. Plant Mol. Biol. Rep 87:395-411. https://doi.org/10.1007/s11103015-0286-1

55. Wang N, Zheng Y, Xin H, Fang L, Li S (2013) Comprehensive analysis of NAC domain transcription factor gene family in Vitis vinifera. Plant Cell Rep 32:61-75. https://doi.org/10.1007/s00299-0121340-y

56. Wang Y, Tang H, DeBarry JD, Tan X, Li J, Wang X, Lee T, Jin H, Marler B, Guo H, Kissinger JD, Andrew HP (2012) MCScanX: a toolkit for detection and evolutionary analysis of gene synteny and collinearity. Nucleic Acids Res 40: e49. https://doi.org/10.1093/nar/gkr1293

57. Yan H, Zhang H, Chen M, Jian H, Baudino S, Caissard JC, Bendahmane Mm, Li S, Zhang T, Zhou N, Qiu X, Wang Q, Tang K (2014) Transcriptome and gene expression analysis during flower blooming in Rosa chinensis 'Pallida'. Gene 544:261. https://doi.org/10.1016/j.gene.2014.02.008

58. Yang X, Wang X, Ji L, Yi Z, Fu C, Ran J, Hu R, Zhou G (2015) Overexpression of a Miscanthus Iutarioriparius NAC gene MINAC5 confers enhanced drought and cold tolerance in Arabidopsis. Plant Cell Rep 34:943-958. https://doi.org/10.1007/s00299-015-1756-2.

59. Yoshihiro N, Kazuo N, Zabta KS, Yoh S, Takashi F, Hiroshi A, Mari N, Kazuo S, Kazuko Y (2003) Interaction between two cis-acting elements, ABRE and DRE, in ABA-dependent expression of Arabidopsis RD29A gene in response to dehydration and high-salinity stresses. Plant $\mathrm{J}$ 34:137-148. https://doi.org/10.1046/j.1365-313X.2003.01708.x 


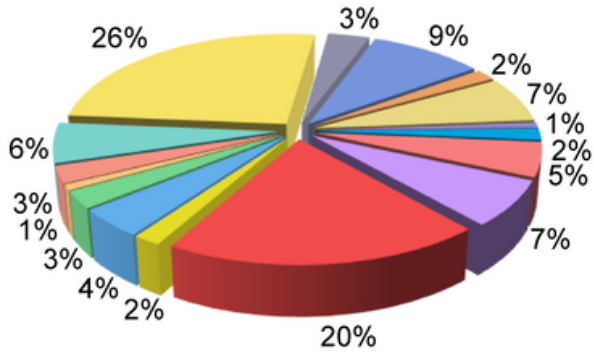

Rosa chinensis

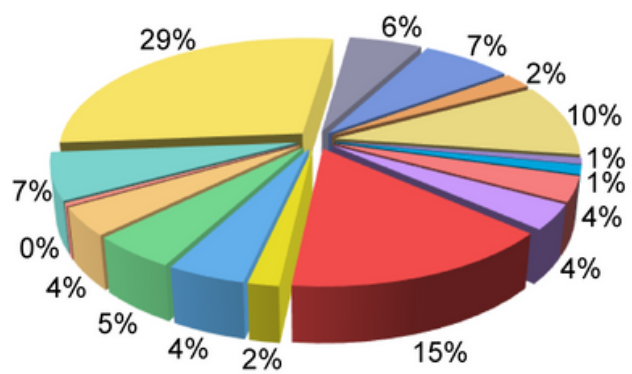

Malus domestica

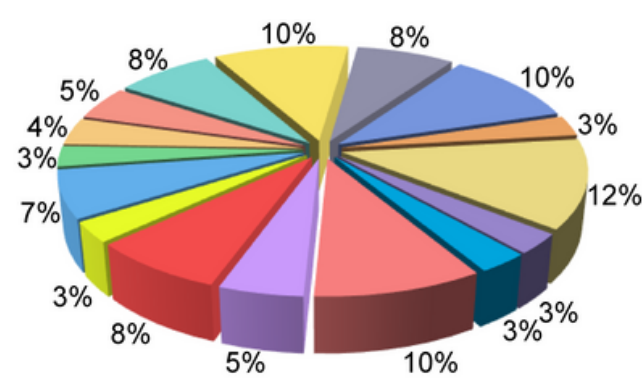

Arabidopsis thaliana

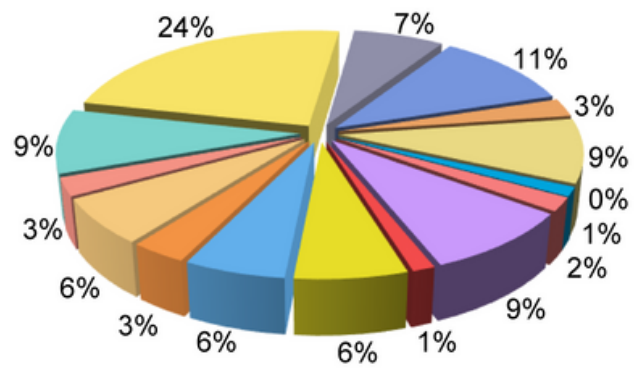

Actinidia chinensis

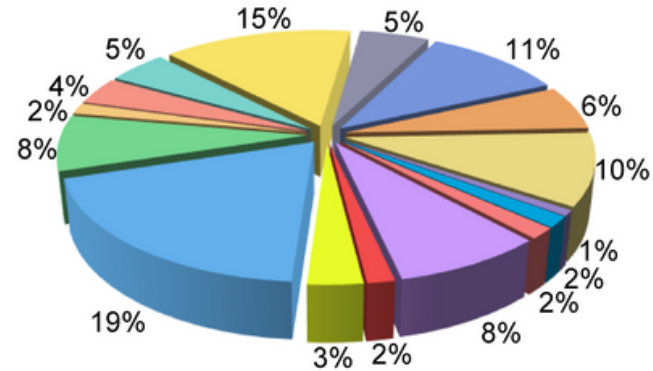

Oryza barthii

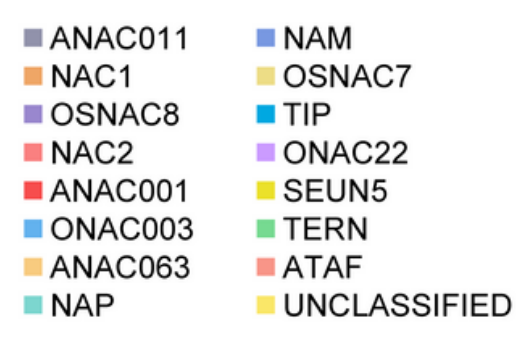

\section{Figure 1}

Percentage of NAC proteins of each subfamily in five plant species. The proteins of rose, Arabidopsis, rice, apple, and kiwifruit were divided into 16 subfamilies, including ANAC011, NAM, NAC1, OSNAC7, OSNAC8, TIP, NAC2, ONAC22, ANAC001, SEUN5, ONAC003, TERN, ANAC063, ATAF, NAP, Unclassified. The percentage represents the proportion of members in each subfamily in the five plant species; different subfamilies are displayed in different colors. 

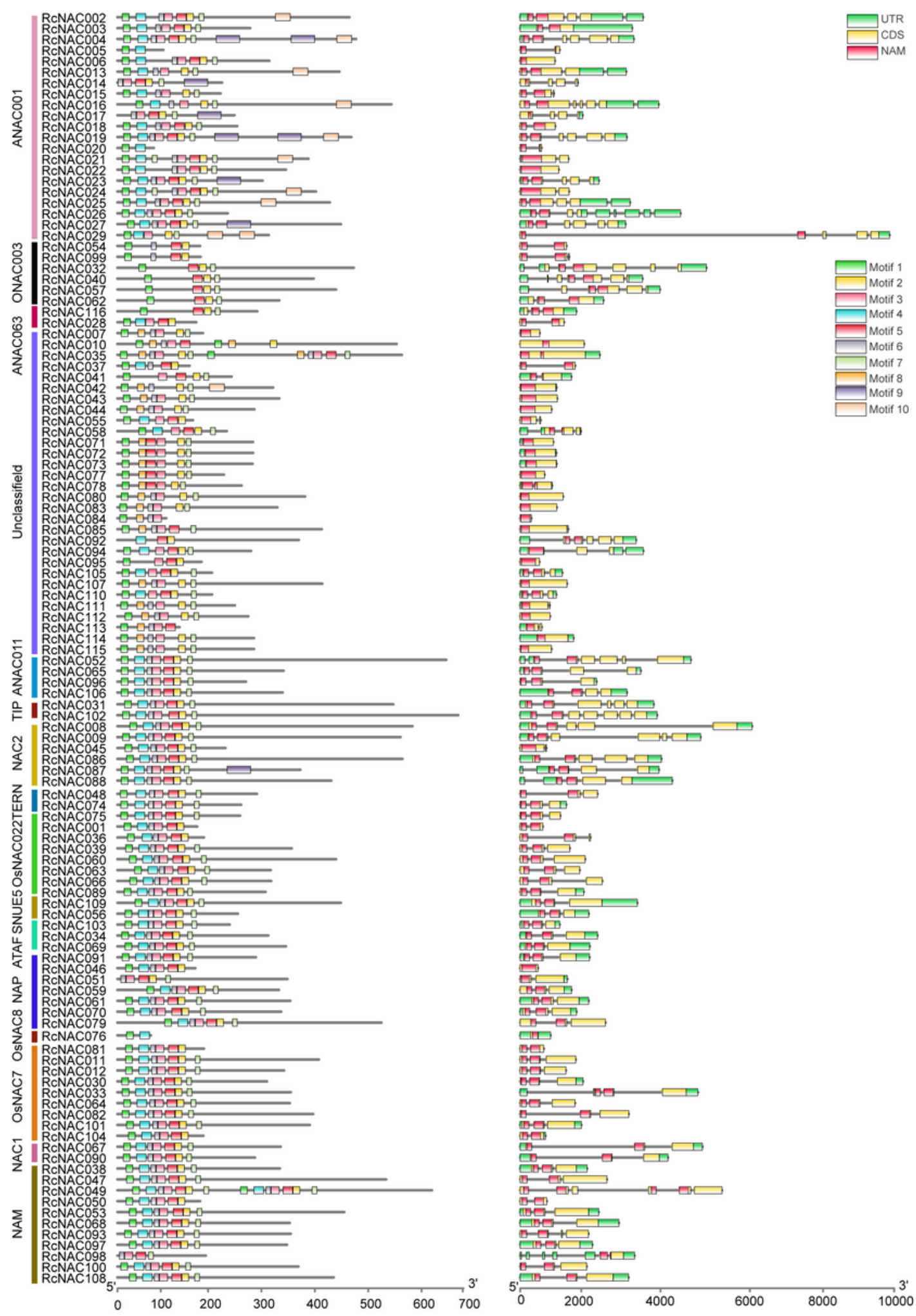

Figure 2

Phylogenetic relationship, motif distribution, and gene structure of RcNACs. A phylogenetic tree was constructed using rose NAC proteins following the neighbor-joining method with 1000 replicates using MEGA 7.0. Colored boxes represent conserved amino acid motifs (1-10) in the RcNACs, and the black line indicates the non-conserved sequences. Exons and introns are indicated by TBtools. The green, 
yellow, and pink boxes represent untranslated region (UTR), coding sequence (CDS), and NAM, respectively.
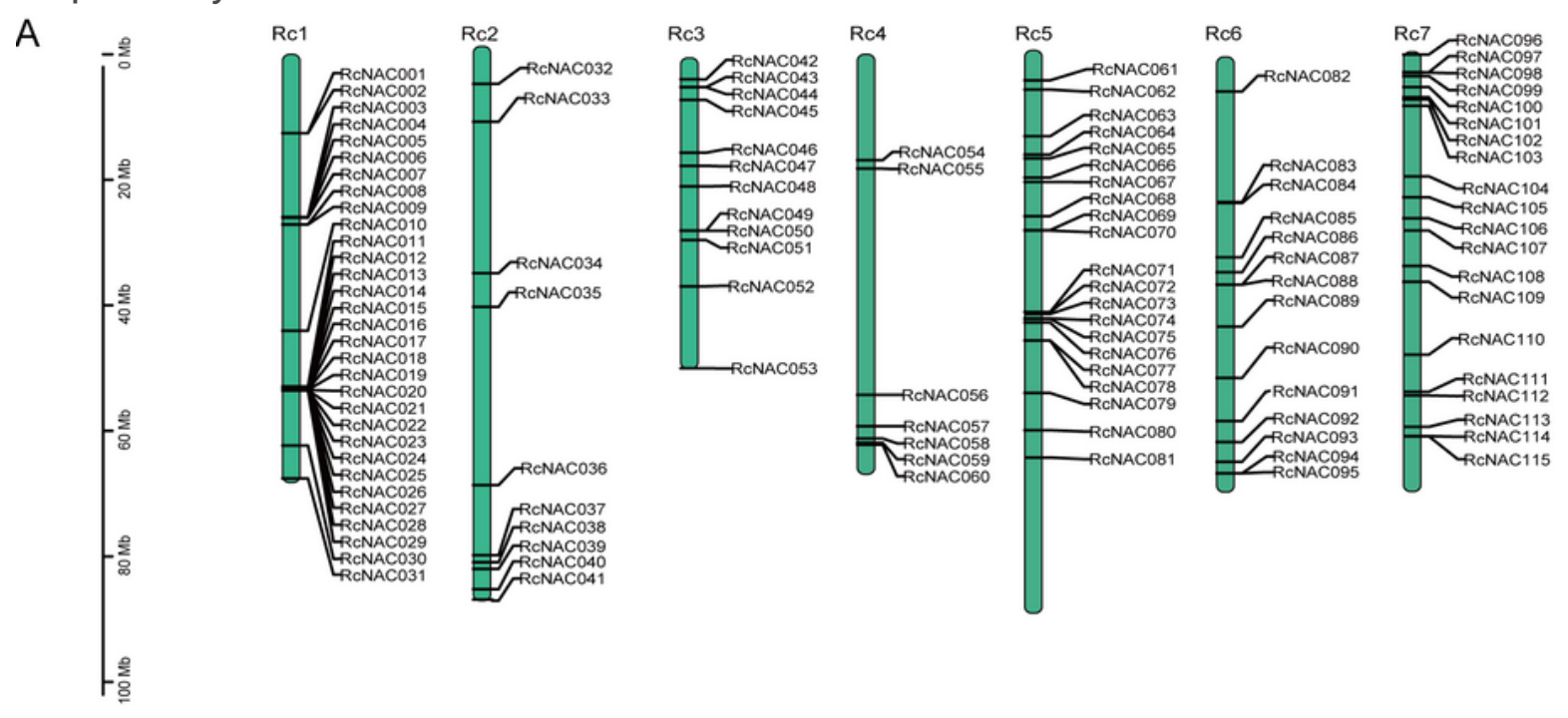

B Rosa chinensis
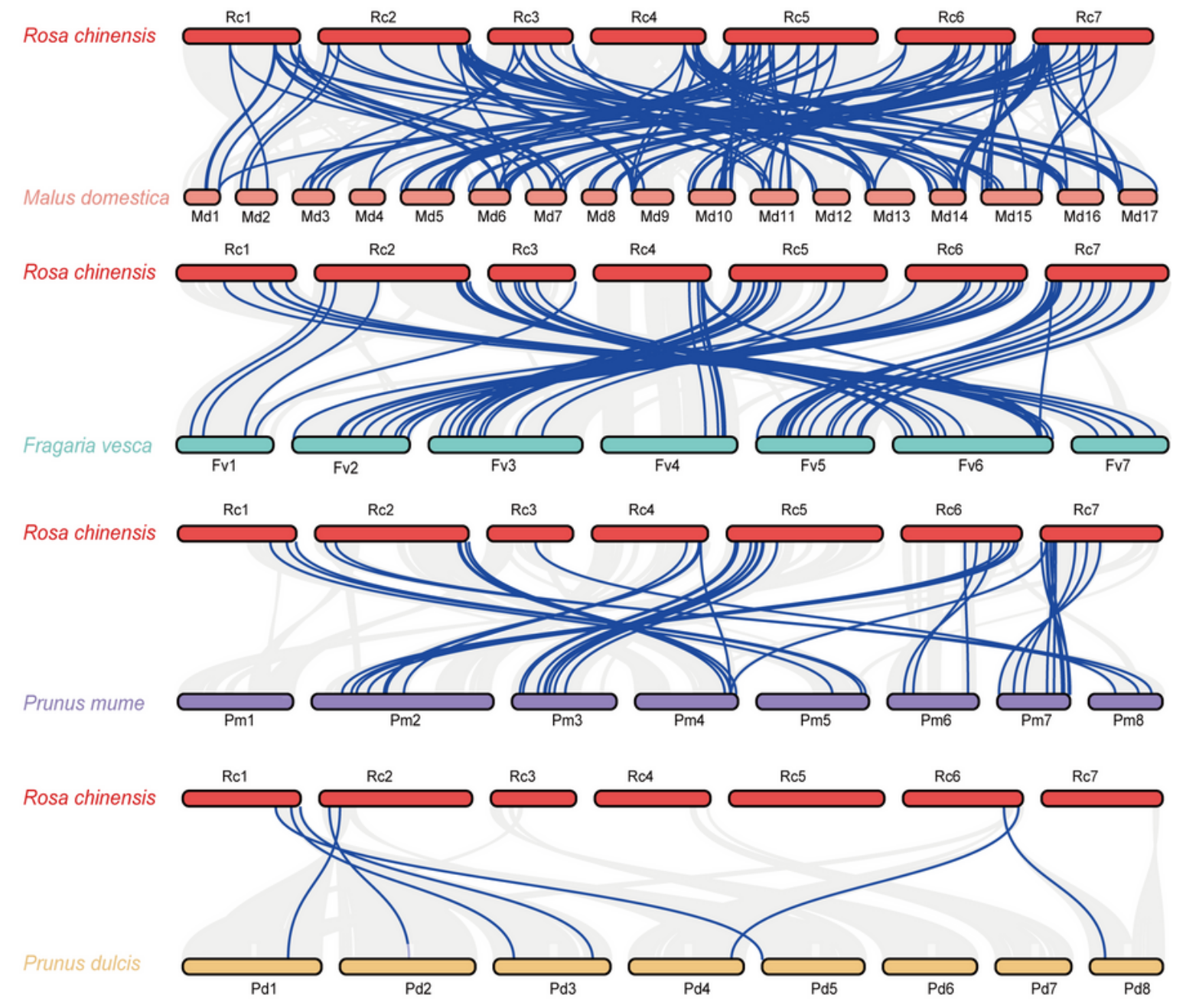

Figure 3

Chromosome location and collinearity analysis of RcNACs. A. RcNACs on the seven chromosomes of rose. The black line between the two gene names indicates tandemly repeated genes. The scale shows the gene position on. The black line indicates the position of the gene on each chromosome. B. 
Synonymous analysis of Rosa chinensis, Malus domestica, Fragaria vesca, Prunus mume, and P. dulcis genes. The gray line in the background indicates the collinear blocks in the genomes of rose and the other four Rosaceae plants, while the blue line emphasizes the isomorphic NAC gene pairs.

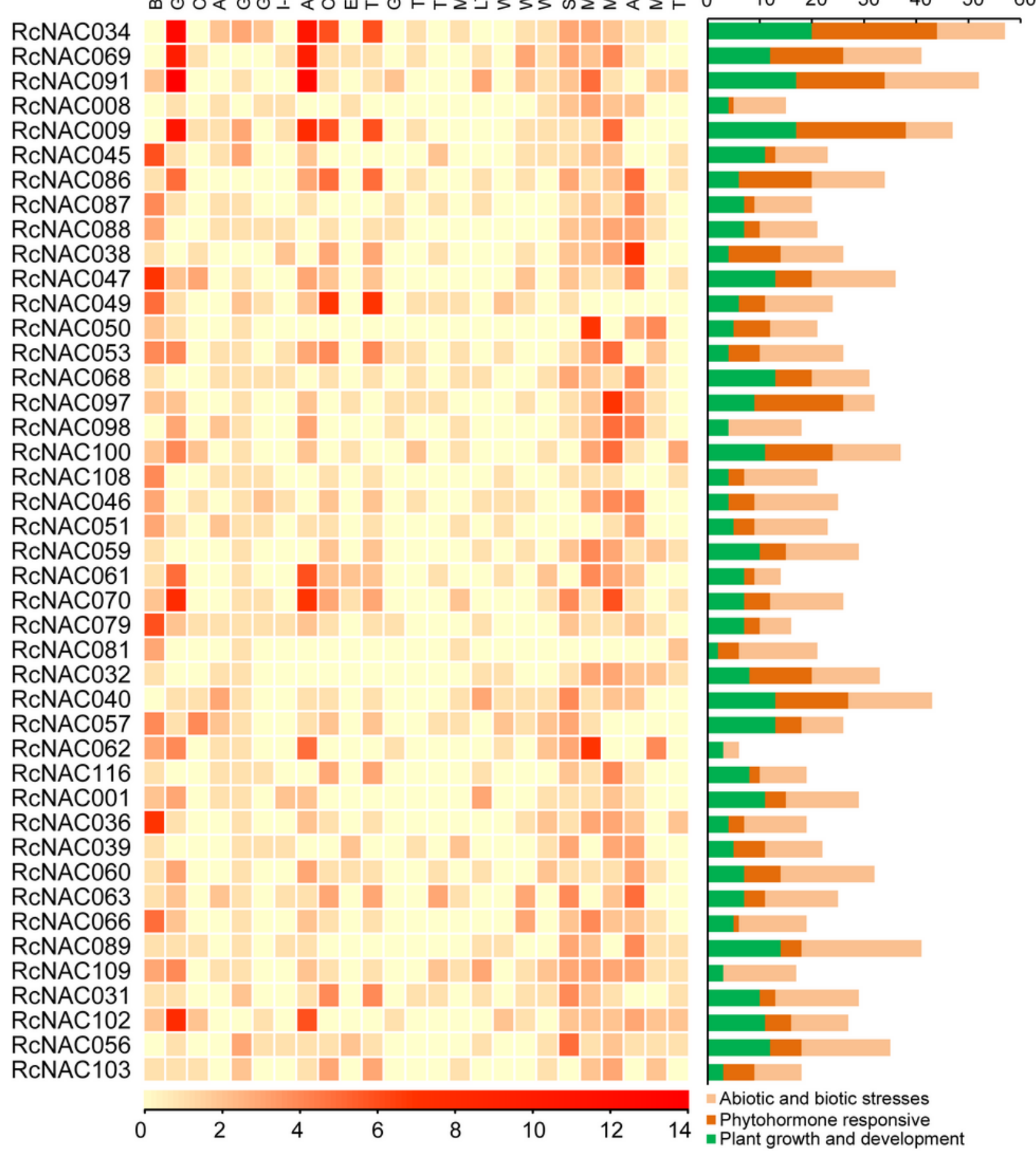

Figure 4

Relative expression levels of RcNACs in different transcriptome datasets. A. Relative expression profiles of RcNACs in different tissues and flower transition stages. Previously reported transcriptome data 
obtained during flower transition (Yu et al. 2019) and from the different organs (Dubois et al. 2011) were used. VM, TM, and FM indicate vegetative meristem, pre-floral meristem, and floral meristem, respectively. B. Relative expression profiles of RcNACs under drought and salt stress. The abscissas ND, MD, and SD represent control, moderate drought, and severe drought, respectively; T0, T2, T4, T7, and T9 represent salt treatment for $0,2,4,7$, and 9 days, respectively; $L$ and $R$ represent roots and leaves, respectively. The drought and salt stress treatments selected for this analysis were defined as described in Li et al. (2021) and Tian et al. (2018), respectivily. The color scale on the right indicates expression values; blue indicates low transcript abundance, and red indicates high transcript abundance.
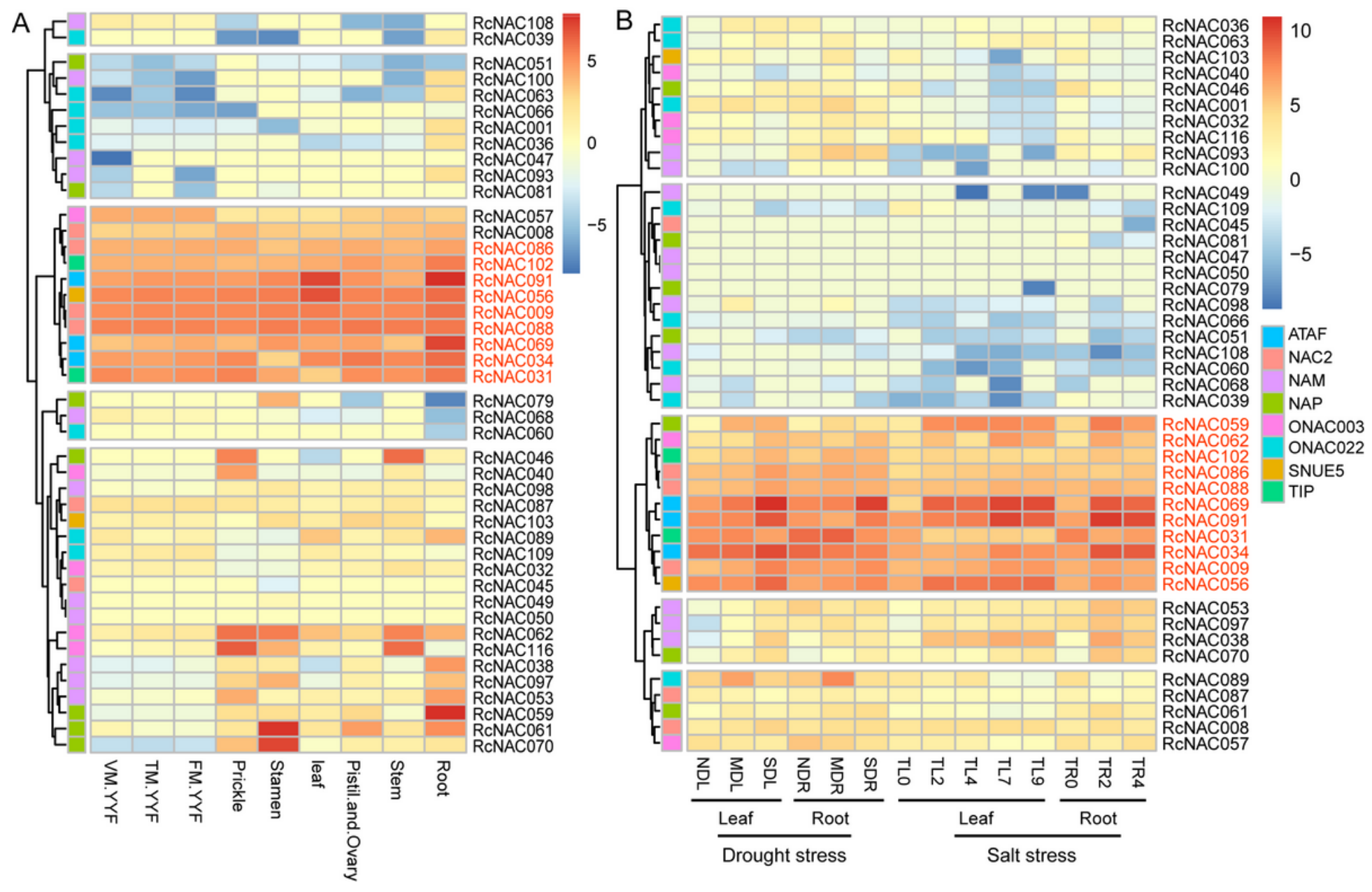

\section{Figure 5}

Analysis of cis-regulatory elements in the RcNAC promoter. The cis-regulatory elements (CREs) in the promoter region ( $1.5 \mathrm{~kb}$ upstream) of RcNACs were predicted by PlantCARE. Heat maps represent different CREs in different RcNACs promoter regions. Different colors indicate three different types of CREs (plant growth and development, plant hormone response, and abiotic and biotic stress). Box 4, Light-inducible promoter induced by ABA and gibberellin (GA) also; G-box, light-responsive; CAT-box, meristem expression; AE-box, light response; GT1-motif, light response; GATA-motif, light-responsive; Ibox, light-responsive; ABRE, ABA- responsive; CGTCA-motif, Methyl jasmonat-responsivs; ERE, Lightinducible promoter also induced by ABA, GA; TGACG-motif, Methyl jasmonate-responsive; GARE-motif, GA-responsive; TGA-element, auxin-responsive; TC-rich repeats, defense and stress-responsive; MBS, MYB 
binding site involved in drought-inducibility; LTR, low-temperature responsive; WUN-motif, wound responsive; W box, WRKY binding site; WRE3-element, high-temperature responsive; STRE-element, stressresponsive element; MYB, dehydration and ABA-responsive; MYC, dehydration and ABA-responsive; ARE, anaerobic induction; Myb-binding, MYB binding site; TCT-motif, part of a light-responsive element.
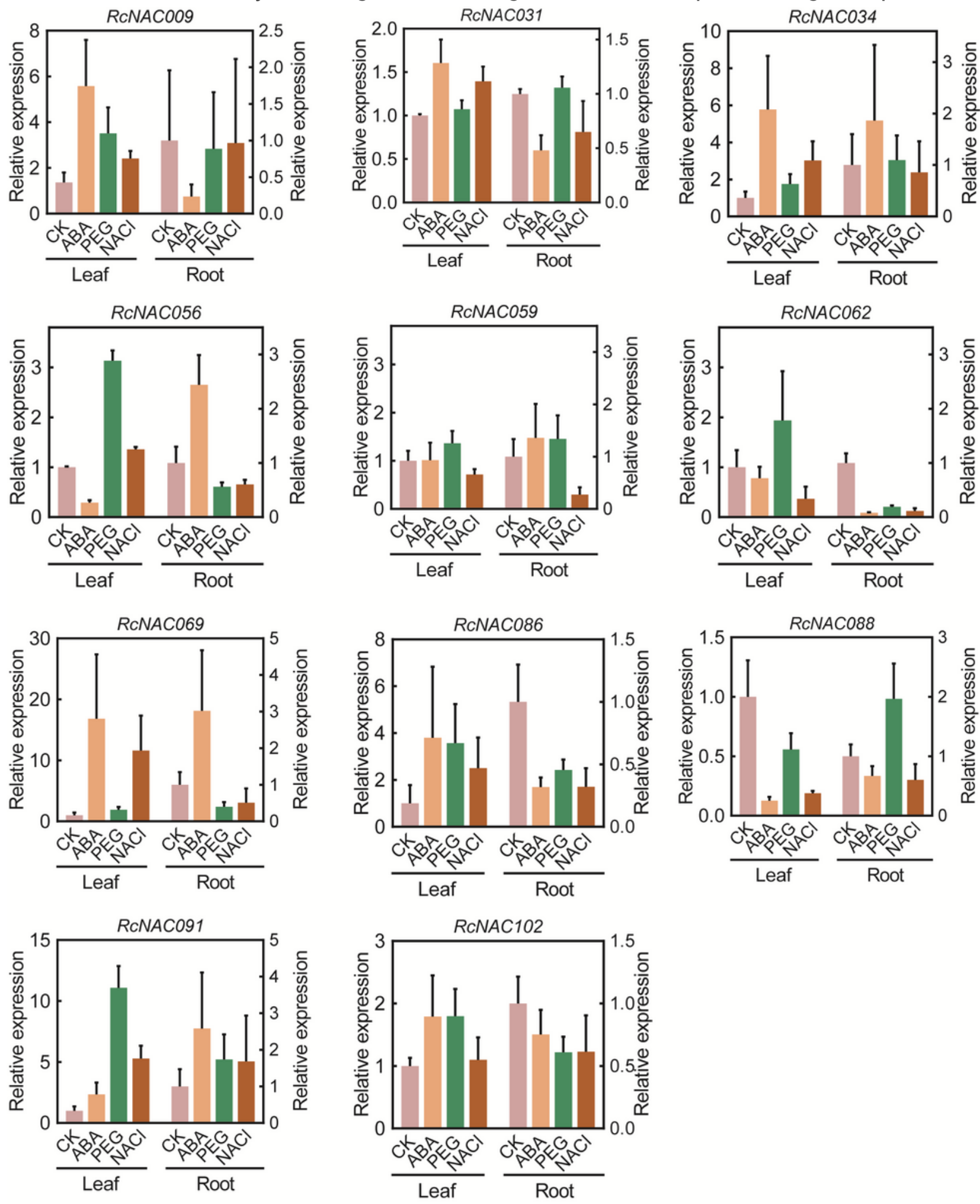

Figure 6 
RT-qPCR analysis of selected stress-related RcNACs. RT-qPCR evaluated 11 stress-related RcNACs. Eightweek-old leaves and roots were treated with $50 \mu \mathrm{M}$ ABA, 10\% PEG 6000, and $200 \mathrm{mM} \mathrm{NaCl}$. Relative expression levels of different RcNACs was normalized to the RcUBI2 gene. Values are means \pm SD of at least three biological experiments.

A

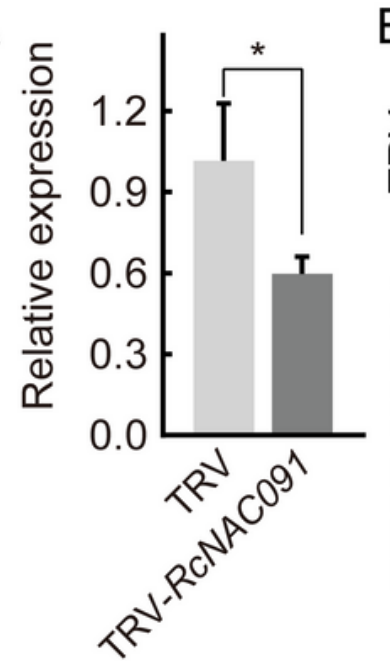

$\mathrm{B}$

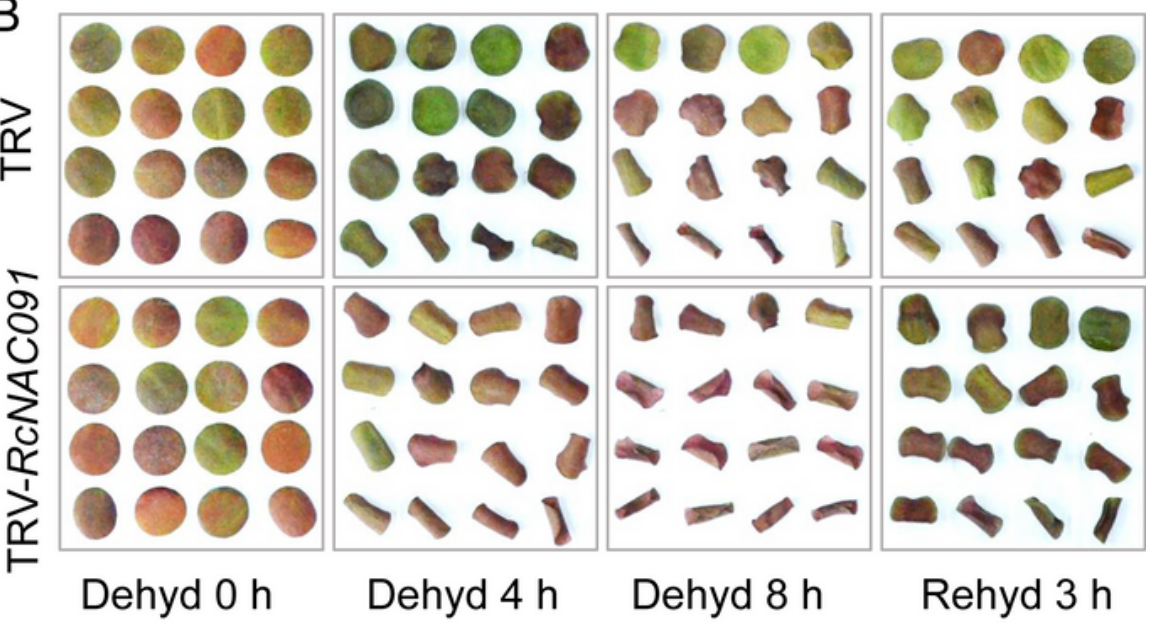

C

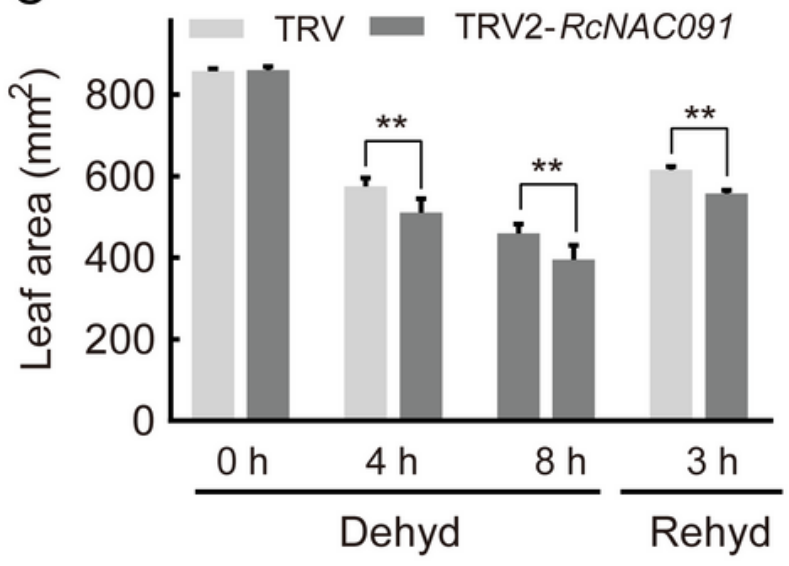

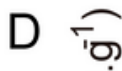

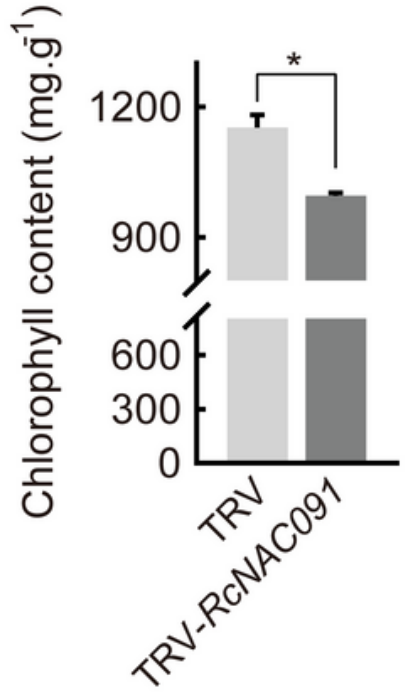

$\mathrm{E}$

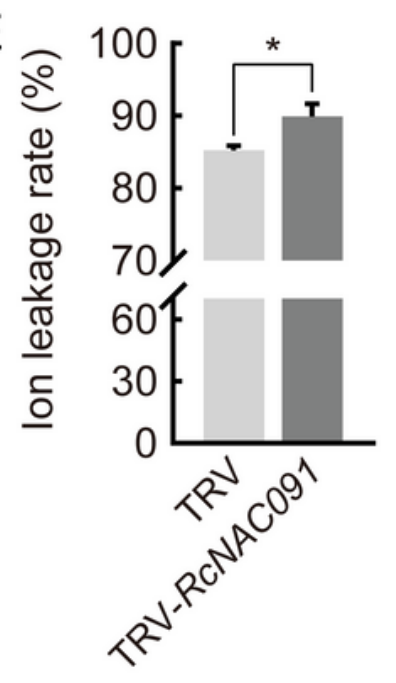

$\mathrm{F}$

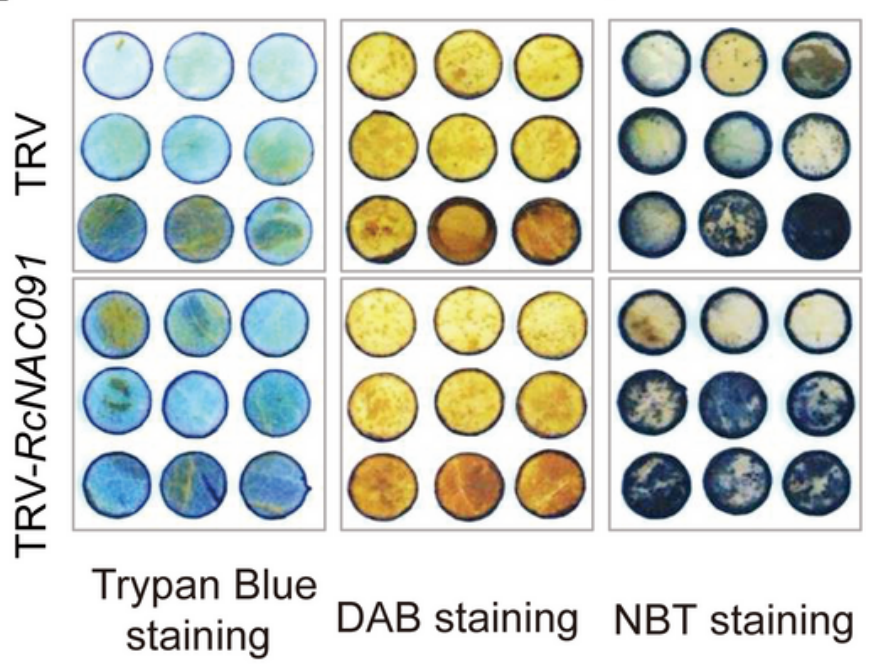

Figure 7 
RcNAC091 gene silencing in rose leaves. A. Expression levels of RcNAC091 transcripts in TRV (Control) and TRV-RcNAC091. The expression levels of determined by RcNAC091 transcripts were normalized to RcUBI2. Data are represented as mean \pm SE (standard error) of three biological replicates. Phenotypic changes (B), disc area (C), chlorophyll content (D), and ion leakage (E) in TRV and TRV-RcNAC091 under dehydration and rehydration conditions. Data are means \pm SE of three biological replicates. Asterisk indicates a significant difference from the control (unpaired Student's t-test; ${ }^{*} P<0.05,{ }^{\star *} P<0.01$ ). (F) Trypan blue, diaminobenzidine, and nitroblue tetrazolium staining of TRV and TRV-RcNAC091 discs under rehydration $3 \mathrm{~h}$ in rose.

\section{Supplementary Files}

This is a list of supplementary files associated with this preprint. Click to download.

- Figure.S1.tif

- TableS1.xIsx

- TableS2.xIsx

- TableS3.xIsx

- TableS4.xIsx

- TableS5.xIsx

- TableS6.xIsx 